\title{
BMJ Open Epidemiology of haemodialysis catheter complications: a survey of 865 dialysis patients from 14 haemodialysis centres in Henan province in China
}

\author{
Kai Wang, Pei Wang, Xianhui Liang, Xiaoqing Lu, Zhangsuo Liu
}

To cite: Wang $K$, Wang $P$, Liang $X$, et al. Epidemiology of haemodialysis catheter complications: a survey of 865 dialysis patients from 14 haemodialysis centres in Henan province in China. BMJ Open 2015;5:e007136. doi:10.1136/bmjopen-2014007136

- Prepublication history for this paper is available online. To view these files please visit the journal online (http://dx.doi.org/10.1136/ bmjopen-2014-007136).

Received 7 November 2014 Revised 2 September 2015 Accepted 20 October 2015

CrossMark

Department of Blood Purification, The First Affiliated Hospital, Zhengzhou University, Institute of Nephrology, Zhengzhou University, Zhengzhou, Henan Province, China

Correspondence to Dr Zhangsuo Liu; liuzhangsuoblood@126.com

\section{ABSTRACT}

Objectives: To investigate the incidence rates and risk factors for catheter-related complications in different districts and populations in Henan Province in China.

Design: Cross-sectional.

Setting: Fourteen hospitals in Henan Province.

Participants: 865 patients with renal dysfunction undergoing dialysis using catheters between October 2013 and October 2014.

Main outcome measures: The main outcome measures were complications, risk factors and patient characteristics. Catheter-related complications included catheter-related infection (catheter exit-site infection, catheter tunnel infection and catheter-related bloodstream infection), catheter dysfunction (thrombosis, catheter malposition or kinking, and fibrin shell formation) and central vein stenosis.

Results: The overall incidence rate was $7.74 / 1000$ catheter-days, affecting $38.61 \%$ of all patients, for catheter infections, 10.58/1000 catheter-days, affecting $56.65 \%$ of all patients, for catheter dysfunction, and $0.68 / 1000$ catheter-days, affecting $8.79 \%$ of all patients, for central vein stenosis. Multivariate analysis showed that increased age, diabetes, primary educational level or below, rural residence, lack of a nephropathy visit before dialysis and pre-established permanent vascular access, not taking oral drugs to prevent catheter thrombus, lower serum albumin levels and higher ferritin levels were independently associated with catheter infections. Rural residence, not taking oral drugs to prevent thrombus, lack of an imaging examination after catheter insertion, non-tunnel catheter type, lack of medical insurance, lack of nephropathy visit before dialysis and pre-established permanent vascular access, left-sided catheter position, access via the femoral vein and lower haemoglobin level were independently associated with catheter dysfunction. Diabetes, lack of nephropathy visit before dialysis and pre-established permanent vascular access, lack of oral drugs to prevent catheter thrombus, left-sided catheter location and higher number of catheter insertions, were independently associated with central vein stenosis.

Conclusions: The rate of catheter-related complications was high in patients with end-stage renal disease in Henan Province. Our finding suggest

\section{Strengths and limitations of this study}

- This is a large study in different settings in the same geographical region.

- Risk factors were comprehensively assessed.

- Some residual confounders were not assessed (medical treatment, nutritional status, psychosocial factors).

- The incidence of catheter-related complications may be underestimated.

that strategies should be implemented to decrease complication rates.

\section{INTRODUCTION}

Dialysis for end-stage renal disease (ESRD) may be achieved using an arteriovenous fistula (AVF) or catheters. ${ }^{1}$ However, the use of catheters is associated with increased allcause mortality, mainly due to catheterrelated infections (CRI). ${ }^{2}{ }^{3}$ According to guidelines, the proportion of patients with ESRD on dialysis who are using an AVF for permanent vascular access should be higher than $65 \%$ and the percentage of patients using a dialysis catheter for permanent vascular access should be lower than $10 \% .^{14}$ However, using a dialysis catheter is still very common. Indeed, according to the annual statistics of the United States Renal Data System (USRDS), ${ }^{5}$ a catheter was used in $62.6 \%$ of dialysis patients for vascular access for their first dialysis treatment in the USA, while only $16 \%$ of patients were using an AVF for vascular access for their first dialysis, while $81 \%$ of patients were using a dialysis catheter as the only method of vascular access or while waiting for AVF maturation.

In addition to CRI, complications from catheter insertion, catheter replacement 
and/or thrombolytic therapy, clots and emboli, exit-site and tunnel inflammation/infection, bacteraemia and sepsis may all occur while the catheter is in place and after catheter removal, increasing mortality risk. ${ }^{6}$ Indeed, CRI will occur in 2.5-5.5 patients per 1000 patient-days or $0.9-2.0$ episodes per patient per year. ${ }^{7}$ Catheter clotting and thrombosis will require plasminogen activator instillation in 3.0 cases per 1000 patientdays and catheter replacement in 1.1 cases per 1000 patient-days. ${ }^{8}$ Thrombi may migrate to the right atrium and superior vena cava, causing pulmonary embolism and superior vena cava syndrome. ${ }^{9}$ Complications threaten patients' safety and treatment efficacy, as well as wasting medical resources: catheter low flow may result in recirculation and under-dialysis; catheter insertion may result in inflammation and an immune reaction; catheter use may result in anaemia, requiring erythropoietin; and frequent problems necessitate more care. ${ }^{6}$ In 2007 alone, Medicare spending on ESRD neared \$24 billion, ${ }^{10}$ with an estimated $\$ 1.8$ billion spent annually on vascular access care. ${ }^{11}$

Currently, there is no proper register of dialysis catheters in China containing epidemiological data about catheter-related complications, risk factors and patient characteristics. Therefore, the aim of this cross-sectional study was to investigate the incidence rates and risk factors for catheter-related complications in different districts and populations in Henan Province in China to help develop effective interventions and public health policies for the most efficient management of patients with ESRD undergoing dialysis using catheters.

\section{SUBJECTS AND METHODS \\ Subjects}

In this cross-sectional study, 865 patients using catheters for dialysis in 14 hospitals in Henan Province between October 2013 and October 2014 were prospectively enrolled. The 14 haemodialysis centres were: the First Affiliated Hospital of Zhengzhou University $(n=125)$, the Third People's Hospital of Zhengzhou City $(n=79)$, the Traditional Chinese Medicine Hospital of Henan Province $(n=65)$, the Traditional Chinese Medicine Hospital of Zhengzhou City ( $n=36$ ), the First Affiliated Hospital of Henan University of Science and Technology $(\mathrm{n}=66)$, Luoyang Eastern Hospital $(\mathrm{n}=56)$, Luoyang Center Hospital $(n=42)$, Huaihe Hospital of Henan University $(n=71)$, the First People's Hospital of Kaifeng City $(n=52)$, the Second People's Hospital of Kaifeng City $(n=47)$, the Puyang Oil Field General Hospital $(n=49)$, the First People's Hospital of Xinxiang City $(n=84)$, the Luohe Center Hospital $(n=42)$ and the Sanmenxia Center Hospital $(\mathrm{n}=51)$.

The inclusion criteria for the hospitals were: (1) at least 10 haemodialysis machines in the haemodialysis centre; (2) at least 50 patients undergoing haemodialysis; and (3) at least 20 patients eligible for the study. The inclusion criteria for the patients were: (1) renal dysfunction and undergoing haemodialysis using catheters for vascular access; (2) willingness to participate in the study, intellectual competence and ability to communicate; and (3) availability of all records of previous vascular access for haemodialysis. Exclusion criteria were: (1) non-cooperation with the investigation; (2) unavailability of previous medical records; (3) aged $<18$ years old; or (4) severe mental disorder or neurological disease.

The study was approved by the ethics committee of the First Affiliated Hospital, Zhengzhou University. Written informed consent was obtained from each participant. Patient records/information was anonymised and de-identified prior to analysis.

\section{Data collection}

Data collected in this study included: (1) the general characteristics of the patients (age, gender, marriage, education, occupation, registered residence, provider payments, underlying disease, frequency of haemodialysis, duration of haemodialysis, and outcomes); (2) catheter data (preparation of vascular access before haemodialysis, reason for the first haemodialysis and the vascular access used, survival rates of non-tunnel haemodialysis catheters (NTHCs) and tunnelled vascular catheters (TVCs), complications, and complications with different types of catheter); and (3) changes in vascular access (frequency of vascular access changes and related reasons, records of catheters used in previous haemodialysis sessions, duration of vascular access, and outcomes). The medical staff in the 14 participating hospitals were trained in catheter monitoring and recording of epidemiological data. Standardised questionnaires were used to record patient information and relevant data collected by the investigators in each hospital. Data were managed centrally at the Henan Blood Dialysis Quality Control Center. Any discrepancy was immediately rectified upon data reception. Data were registered by trained registrars.

\section{Insertion of dialysis catheters}

Experienced physicians (attending specialists in kidney diseases with a minimum of 1 year of experience and 100 cases of catheter insertion) from the nephrology department followed aseptic practices in the operating room. The venipuncture site was prepared by disinfection with alcohol three times and then with betadine (a common disinfectant used in all 14 hospitals). There was no prophylactic use of antibiotics. The first choice for catheter insertion was the right internal jugular vein, followed by the right external jugular vein, left internal/ external jugular vein, subclavian vein and femoral vein in that order. Central vein catheterisation was performed using the Seldinger technique (a subcutaneous tunnel was needed for TVCs). If an NTHC was required, single needle double-lumen catheters (ARROW: Arrow International, Asheboro, North Carolina, USA; DIALL: DIALL Medical Technology, Zhengzhou, China; ABLE: 
Guangdong Baihe Medical Technology, Foshan, China; TYCO: Covidien, USA) were used, while the Quinton Palindrome (Covidien), Quinton Permcath (Covidien), Arrow Cannon II Plus (Arrow International) and Bard HemoSplit (CR Bard, Tempe, Arizona, USA) were used for permanent indwelling catheters. The length and size of catheters depended on the age and height of the patient and the site of the indwelling catheter. The type of catheter was decided by the hospital. Local infiltration anaesthesia was most commonly used, with general anaesthesia reserved for children or uncooperative patients. The patient was evaluated before indwelling catheters were inserted. Doppler ultrasonography-guided puncture or venography-assisted indwelling catheter insertion was used in patients with vascular malformation, stenosis and occlusion of the central vein, or a pacemaker.

\section{Haemodialysis and catheter care}

Patients were dialysed 2-4 times per week for $4-5 \mathrm{~h}$ per treatment. Blood flow was $5 \mathrm{~mL} / \mathrm{kg}$ per min, and dialysate flow was $300-500 \mathrm{~mL} / \mathrm{min}$. Hollow-fibre dialysers were selected according to patient body size (Fresenius, Gambro or Nipro haemodialysis machines). A standard bicarbonate buffer was used as dialysate buffer. Microbiological water purity was checked monthly to ensure compliance with tight chemical and microbiological standards $(<0.1 \mathrm{cfu} / \mathrm{mL}$ and $<0.03 \mathrm{EU} / \mathrm{mL})$. Haemodialysis catheters were handled only during dialysis sessions with no irrigation between treatments. According to standard nursing procedures, the catheter exit site and the two ports were cleaned with povidone iodine solution, applied with a Biopatch (chlorhexidine-impregnated dressing), and covered with a permeable dressing at the end of each dialysis session. Each port of the catheter was filled with $5000 \mathrm{U} / \mathrm{mL}$ of heparin solution according to the manufacturer's recommendation. Each time before the catheter was opened, the exit site of the catheter was carefully checked for any redness, swelling, bleeding or exudate, and patients were asked to report any fever episodes. In addition, the temperature of each patient was measured twice during dialysis. This process was repeated for each haemodialysis treatment.

\section{Definitions of dialysis catheter-related complications Catheter-related infections}

Catheter exit-site infection was diagnosed if pus, redness, induration or tenderness within $2 \mathrm{~cm}$ around the catheter exit site was present, or pus secretion culture was positive. ${ }^{12}$ Suspicious signs were the presence of pus, redness, induration or tenderness at the catheter exit site, but negative pus secretion culture. There were no signs of infection in other sites such as gauze and sutures.

Catheter tunnel infection was diagnosed if pus, redness, tenderness and/or induration $(>2 \mathrm{~cm})$ along the catheter tunnel was present, with a positive bacteria culture from secretions. Suspicious signs were presence of pus, redness, induration and tenderness along the catheter tunnel, without a positive culture from secretions or infections in other loci.

Catheter-related bloodstream infection (CRBSI) was diagnosed if the same microorganism was grown from peripheral blood culture and catheter tip culture at least once, or the count of colonies cultured from catheter lock solution was threefold or more greater than the count from peripheral blood culture. Suspicious signs were positive blood cultures obtained from catheter lock solution and/or peripheral blood in a symptomatic patient with no clinical evidence of infections in other loci.

\section{Catheter dysfunction}

Catheter dysfunction was identified when at least one of the following criteria was met: (1) peak blood flow $<200 \mathrm{~mL} / \mathrm{min}$ for at least $30 \mathrm{~min}$; (2) mean blood flow $<250 \mathrm{~mL} / \mathrm{min}$ during two consecutive dialysis sessions; or (3) unable to initiate dialysis due to inadequate blood flow even after timely treatment (the criteria could be lower for children or patients with low body weight).

\section{Thrombosis}

Intrinsic catheter thrombosis occurs when a thrombus is formed and attaches to the inner or outer surface of the catheter (including thrombi in the lumen or at the tip of the catheter). Extrinsic catheter thrombosis occurs when a thrombus is caused by the presence of a catheter in the atrium or central vein.

\section{Catheter malposition or kinking}

Catheter malposition or kinking occurs when the position of the catheter tip is in the wrong place or moves after being placed, or the catheter is curved or folded.

\section{Fibrin shell}

A fibrin shell is a membrane substance consisting of endothelial cells, smooth muscle cells and collagen wrapping around the central venous catheter, which can happen within $24 \mathrm{~h}$ after catheter insertion. ${ }^{12}$ Fibrin shells can cause several complications including catheter dysfunction, infection, stenosis of the central vein and pulmonary embolism after catheter removal. The presence of fibrin shells was evaluated by digital subtraction angiography.

\section{Central vein stenosis}

Damage caused by the dialysis catheter after insertion, along with comorbidities, can induce stenosis of the central vein and thus cause obstruction syndrome, which in turn results in a series of symptoms and signs including swelling of the limbs, head and neck after stenosis of the vena cava system, ulcers and infection in the limbs, and cerebral oedema in severe cases. The presence of clinical symptoms, Doppler ultrasound examinations and angiography are required for diagnosis, with angiography confirming the location and extent of the obstruction. 


\section{Statistical analysis}

SPSS V.17.0 (SPSS Inc., Chicago, Illinois, USA) was used for data management and analysis. The general characteristics of the patients are presented as means and standard deviations or frequencies and percentages. Incidence rates per 1000 catheter-days were calculated for catheter complications. Categorical variables were compared using a $\chi^{2}$ test. Logistic regression was used to investigate the risk factors influencing the outcomes of catheter infection, catheter dysfunction and central vein stenosis, which were the dependent variables; the independent variables were patient characteristics (including treatment) and parameters surrounding catheter insertion. The independent variables investigated were age $(\leq 17,18-44,45-59, \geq 60$ years), gender (female vs male), registered residency (urban vs rural), marital status (unmarried vs married), primary disease (nondiabetes vs diabetes), level of education (university, middle school, primary school or below), occupation (farmer and unemployed vs others), source of payment for medical costs (self-paying, New Rural Cooperative Medical System, medical insurance), serum albumin level $(>40,35-40,<35 \mathrm{~g} / \mathrm{L})$, serum ferritin level $(<100$, $100-800,>800 \mathrm{ng} / \mathrm{mL})$, haemoglobin level $(<60,60-90$, $>90 \mathrm{~g} / \mathrm{L})$, duration of haemodialysis $(\geq 48,24-48,<24$ months), haemodialysis frequency ( $3 /$ week, $5 / 2$ weeks, $2 /$ week, 3/2 weeks, $\leq 1 /$ week), nephropathy visit before dialysis treatment (yes vs no), pre-established permanent vascular access (yes vs no), taking drugs orally to prevent catheter thrombus such as aspirin, clopidogrel or warfarin (yes vs no), imaging examination after catheter insertion (yes vs no), type of haemodialysis catheter (NTHC vs TVC), catheter access vein (femoral, internal jugular, external jugular, subclavian), catheter location (left vs right side) and catheter insertion time $(\geq 3,2$, $1 \mathrm{~min})$. The results are presented as ORs and 95\% CIs. Two-tailed $\mathrm{p}$ values $<0.05$ were considered statistically significant.

\section{RESULTS}

\section{Characteristics of patients}

Table 1 presents the characteristics of 865 patients included in the study between October 2013 and October 2014. A total of 564 had indwelling NTHCs for 136.2 \pm 67.4 days and 385 had TVCs for 1052.3 \pm 371.3 days. Patients were aged $49.53 \pm 18.42$ years. There were $495(57.2 \%)$ males and 370 females (42.8\%). Among all patients, $386(44.6 \%)$ were receiving help from the New Rural Cooperative Medical System, 319 $(36.9 \%)$ had medical insurance, and $160(18.5 \%)$ were paying for their medical care themselves. The three most common reasons for ESRD were diabetic kidney disease $(n=236,27.3 \%)$, primary nephropathy $(n=143$, $16.5 \%)$ and hypertensive nephropathy $(\mathrm{n}=96,11.1 \%)$. At the time of the study, patients had undergone dialysis for a median of 26.5 months (range: 15 days to 18 years). Haemodialysis frequency was twice a week in
Table 1 Patient characteristics

\begin{tabular}{|c|c|}
\hline Characteristics & Values $(\mathrm{N}=865)$ \\
\hline NTHCs & 564 \\
\hline TVCs & 385 \\
\hline \multicolumn{2}{|l|}{ Placement duration (days) } \\
\hline NTHCs & $136.2 \pm 67.4$ \\
\hline TVCs & $1052.3 \pm 371.3$ \\
\hline \multicolumn{2}{|l|}{ Permanent catheter } \\
\hline Quinton Palindrome & $103(24.4 \%)$ \\
\hline Quinton Permcath & $91(21.6 \%)$ \\
\hline Arrow Cannon II Plus & $101(29.1 \%)$ \\
\hline Bard HemoSplit & $90(24.9 \%)$ \\
\hline Age (years) & $49.53 \pm 18.42$ \\
\hline$\leq 18$ & $126(14.6 \%)$ \\
\hline $19-44$ & $212(24.5 \%)$ \\
\hline $45-59$ & $236(27.3 \%)$ \\
\hline$\geq 60$ & 291 (33.6\%) \\
\hline \multicolumn{2}{|l|}{ Gender } \\
\hline Male & $495(57.2 \%)$ \\
\hline Female & $370(42.8 \%)$ \\
\hline \multicolumn{2}{|l|}{ Marital status } \\
\hline Married & $487(56.3 \%)$ \\
\hline Single & 285 (32.9\%) \\
\hline Widowed or divorced & $93(10.8 \%)$ \\
\hline \multicolumn{2}{|l|}{ Education } \\
\hline University & $152(17.6 \%)$ \\
\hline Middle school & $481(55.6 \%)$ \\
\hline Primary school or below & $232(26.8 \%)$ \\
\hline \multicolumn{2}{|l|}{ Occupation } \\
\hline Farmer & $352(40.7 \%)$ \\
\hline Worker & $124(14.3 \%)$ \\
\hline Office worker & $95(11.0 \%)$ \\
\hline Retired & $165(19.1 \%)$ \\
\hline Unemployed & $129(14.9 \%)$ \\
\hline \multicolumn{2}{|l|}{ Registered residence } \\
\hline Rural & $497(57.5 \%)$ \\
\hline Urban & $368(42.5 \%)$ \\
\hline \multicolumn{2}{|l|}{ Medical costs } \\
\hline $\begin{array}{l}\text { New Rural Cooperative Medical } \\
\text { System }\end{array}$ & $386(44.6 \%)$ \\
\hline Medical insurance & $319(36.9 \%)$ \\
\hline Self-paying & $160(18.5 \%)$ \\
\hline \multicolumn{2}{|l|}{ Primary disease } \\
\hline Diabetic kidney disease & $236(27.3 \%)$ \\
\hline Primary nephropathy & $143(16.5 \%)$ \\
\hline Hypertensive nephropathy & $96(11.1 \%)$ \\
\hline Acute renal failure & $44(5.1 \%)$ \\
\hline Obstructive nephropathy & $72(8.3 \%)$ \\
\hline Renal tubular interstitial disease & $62(7.2 \%)$ \\
\hline Drug-induced renal damage & 39 (4.5\%) \\
\hline Myeloma & $24(2.8 \%)$ \\
\hline Others & $103(11.9 \%)$ \\
\hline $\begin{array}{l}\text { Haemodialysis duration, median } \\
\text { (range) }\end{array}$ & $\begin{array}{l}26.5 \text { months ( } 15 \text { days to } \\
18 \text { years) }\end{array}$ \\
\hline \multicolumn{2}{|l|}{ Haemodialysis frequency } \\
\hline Twice per week & $375(43.4 \%)$ \\
\hline Three times per week & $182(21.0 \%)$ \\
\hline Three times every 2 weeks & $121(14.0 \%)$ \\
\hline Five times every 2 weeks & $145(16.8 \%)$ \\
\hline
\end{tabular}

$375(43.4 \%)$, three times a week in $182(21.0 \%)$, three times every 2 weeks in $121(14.0 \%)$ and five times every 2 weeks in $145(18.6 \%)$ patients. 


\section{Complications}

The overall incidence of catheter infection was 7.74/ 1000 catheter-days, affecting $38.61 \%$ of all patients in the study. The overall incidence rate of catheter dysfunction was $10.58 / 1000$ catheter-days, affecting $56.65 \%$ of patients. The overall incidence rate of central vein stenosis was $0.68 / 1000$ catheter-days, affecting $8.79 \%$ of patients.

\section{Univariate analysis of factors for catheter-related complications}

Increased age $(\leq 17,18-44,45-59, \geq 60 ; \mathrm{OR}=0.427,95 \%$ CI 0.242 to $0.631, \mathrm{p}=0.041$ ), diabetes (non-diabetes vs diabetes; $\mathrm{OR}=0.416,95 \%$ CI 0.262 to $0.826, \mathrm{p}<0.01$ ), primary school education or below (primary school or below vs university; OR=2.405, 95\% CI 1.373 to 4.214, $\mathrm{p}=0.002$ ), rural registered residence (urban vs rural; $\mathrm{OR}=0.250,95 \%$ CI 0.157 to $0.398, \mathrm{p}<0.01$ ), lack of a nephropathy visit before dialysis treatment (yes vs no; $\mathrm{OR}=0.110,95 \%$ CI 0.064 to $0.189, \mathrm{p}<0.01$ ), lack of preestablished permanent vascular access (yes vs no; $\mathrm{OR}=0.242,95 \%$ CI 0.154 to $0.381, \mathrm{p}<0.01$ ), not taking oral drugs to prevent catheter thrombus (yes vs no; $\mathrm{OR}=0.218,95 \%$ CI 0.125 to $0.379, \mathrm{p}<0.01$ ), low serum albumin level $(>40,35-40,<35 \mathrm{~g} / \mathrm{L} ; \mathrm{OR}=0.400,95 \%$ CI 0.300 to $0.534, \mathrm{p}<0.01)$ and high serum ferritin level $(<100,100-800,>800 \mathrm{ng} / \mathrm{mL} ; \mathrm{OR}=1.857,95 \%$ CI 1.375 to $2.508, \mathrm{p}<0.01)$ were associated with catheter infection (table 2).

A rural registered residence (urban vs rural; $\mathrm{OR}=0.218$, 95\% CI 0.137 to $0.345, \mathrm{p}<0.01$ ), lack of medical insurance (New Rural Cooperative Medical System vs medical insurance; $\mathrm{OR}=3.762,95 \%$ CI 0.517 to $6.319, \mathrm{p}=0.047$; selfpaying vs medical insurance; OR=6.412, 95\% CI 3.309 to $12.076, \mathrm{p}=0.017$ ), lack of a nephropathy visit before dialysis treatment (yes vs no; $\mathrm{OR}=0.056,95 \%$ CI 0.033 to 0.097, $\mathrm{p}<0.01$ ), lack of pre-established permanent vascular access (yes vs no; OR $=0.114,95 \%$ CI 0.063 to 0.208 , $\mathrm{p}<0.01$ ), NTHC catheter type (NTHC vs TVC; OR=1.793, 95\% CI 1.510 to 2.231, $\mathrm{p}=0.031$ ), left-sided catheter position (right vs left; OR $=0.067,95 \%$ CI 0.034 to 0.130 , $\mathrm{p}<0.01$ ), lack of imaging examination after catheter insertion (no vs yes; $\mathrm{OR}=2.827$, 95\% CI 1.804 to 4.430, $\mathrm{p}<0.01$ ), femoral vein catheter access (internal jugular vein vs femoral vein; $\mathrm{OR}=0.126,95 \%$ CI 0.044 to 0.361 , $\mathrm{p}<0.01$; external jugular vein vs femoral vein; $\mathrm{OR}=0.06$, 95\% CI 0.017 to $0.216, \mathrm{p}<0.01$; subclavian vein vs femoral vein; $\mathrm{OR}=0.094,95 \%$ CI 0.027 to $0.332, \mathrm{p}<0.01$ ), not taking oral drugs to prevent catheter thrombus (yes vs no; $\mathrm{OR}=0.100,95 \%$ CI 0.059 to $0.168, \mathrm{p}<0.01)$ and lower haemoglobin level $(<60,60-90,>90 \mathrm{~g} / \mathrm{L} ; \mathrm{OR}=1.421,95 \%$ CI 1.318 to $1.558, \mathrm{p}<0.01)$ were associated with catheter dysfunction (table 2).

Diabetes (non-diabetes vs diabetes; OR $=0.692,95 \%$ CI 0.148 to $0.871, \mathrm{p}<0.01$ ), lack of a nephropathy visit before dialysis treatment (yes vs no; $\mathrm{OR}=0.249,95 \%$ CI 0.148 to $0.420, \mathrm{p}<0.01$ ), lack of pre-established permanent vascular access (yes vs no; OR=0.225, 95\% CI 0.140 to $0.362, \mathrm{p}<0.01$ ), left-sided catheter position (right vs left; $\mathrm{OR}=0.325,95 \%$ CI 0.204 to $0.516, \quad \mathrm{p}<0.01)$, increased number of indwelling catheters $(\geq 3,2,1$; $\mathrm{OR}=2.471,95 \%$ CI 1.818 to $3.360, \mathrm{p}<0.01)$ and not taking oral drugs to prevent catheter thrombus (yes vs no; $\mathrm{OR}=0.189,95 \%$ CI 0.089 to $0.362, \mathrm{p}<0.01$ ) were associated with central vein stenosis (table 2).

\section{Multivariate analysis of factors for catheter-related complications}

The results of multivariate analysis are presented in table 3.

Analysis of the relationship between independent variables and catheter infection by logistic regression showed that there were independent relationships between catheter infection and patient age $(\mathrm{OR}=0.351,95 \%$ CI 0.136 to 0.674 ; older patients had a higher catheter infection risk), the patient's registered residency $(\mathrm{OR}=0.250,95 \%$ CI 0.120 to 0.520 ; the catheter infection risk for rural registered residents was higher than for urban registered residents), primary disease ( $\mathrm{OR}=0.379,95 \%$ CI 0.176 to 0.818 ; the catheter infection risk for patients with primary diabetes was higher than for patients without primary diabetes) and educational level (OR=10.757, 95\% CI 3.637 to 31.817 for primary school or below versus university; patients with higher educational levels had a lower catheter infection risk). Factors protective against catheter infection were nephropathy visit before dialysis treatment and establishing long-term vascular access in advance $(\mathrm{OR}=0.22,95 \%$ CI 0.096 to 0.502 and $\mathrm{OR}=0.401,95 \%$ CI 0.193 to 0.832 , respectively), taking drugs orally to prevent catheter thrombus $(\mathrm{OR}=0.611,95 \%$ CI 0.404 to 0.923; the catheter infection risk for patients not taking drugs orally to prevent catheter thrombus was higher than for patients taking drugs orally to prevent catheter thrombus), serum protein level ( $\mathrm{OR}=0.142,95 \%$ CI 0.085 to 0.237$)$ and serum ferritin level $(\mathrm{OR}=2.162,95 \%$ CI 1.412 to 23.308) (patients with lower serum protein levels and higher serum ferritin levels had higher risks of catheter infection). Analysis of the relationship between independent variables and catheter dysfunction by logistic regression showed that there were independent relationships between catheter dysfunction and the following factors: registered residency $(\mathrm{OR}=0.021,95 \%$ CI 0.004 to 0.101 ; the risk of catheter dysfunction for rural patients was higher than for urban patients), taking drugs orally to prevent catheter thrombus $(\mathrm{OR}=0.106,95 \%$ CI 0.041 to 0.274 ; the risk of catheter dysfunction for patients not taking drugs was obviously higher than for patients taking drugs), imaging examination after catheter insertion $(\mathrm{OR}=2.631,95 \%$ CI 1.293 to 5.354; the risk for patients without imaging examination after catheter insertion was significantly higher), catheter type (OR=3.493, 95\% CI 1.358 to 8.983; NTHCs had higher risk than TVCs), method of payment (OR=13.416, 95\% CI 2.541 to 70.827; patients with medical insurance had lower risk than selfpaying patients). Nephropathy visit before dialysis treatment and establishing long-term vascular access in 
Table 2 Univariate logistic regression of factors influencing the development of dialysis catheter-related complications

\begin{tabular}{|c|c|c|c|c|c|c|c|c|c|}
\hline \multirow[b]{2}{*}{ Variables } & \multicolumn{3}{|c|}{ Catheter infection } & \multicolumn{3}{|c|}{ Catheter dysfunction } & \multicolumn{3}{|c|}{ Central vein stenosis } \\
\hline & OR & $95 \% \mathrm{Cl}$ & p Value & OR & $95 \% \mathrm{Cl}$ & p Value & $\overline{\text { OR }}$ & $95 \% \mathrm{Cl}$ & p Value \\
\hline Age & 0.427 & 0.242 to 0.631 & 0.041 & 1.157 & 0.745 to 1.797 & 0.516 & 1.469 & 0.934 to 2.310 & 0.096 \\
\hline Gender & 0.752 & 0.491 to 1.152 & 0.19 & 1.046 & 0.680 to 1.610 & 0.837 & 1.132 & 0.723 to 1.772 & 0.588 \\
\hline Primary disease & 0.416 & 0.262 to 0.826 & $<0.01$ & 0.738 & 0.266 to 1.613 & 0.125 & 0.692 & 0.148 to 0.871 & $<0.01$ \\
\hline Marriage & 1.109 & 0.701 to 1.754 & 0.658 & 1.318 & 0.819 to 2.122 & 0.256 & 1.377 & 0.854 to 2.221 & 0.189 \\
\hline \multicolumn{10}{|l|}{ Education } \\
\hline University & & & $<0.01$ & & & 0.225 & & & 0.150 \\
\hline Middle school & 0.669 & 0.406 to 1.104 & 0.116 & 0.668 & 0.411 to 1.086 & 0.104 & 0.669 & 0.406 to 1.104 & 0.116 \\
\hline Primary school or below & 2.405 & 1.373 to 4.214 & 0.002 & 0.962 & 0.538 to 1.719 & 0.895 & 0.392 & 0.204 to 0.753 & 0.072 \\
\hline Occupation & 1.380 & 0.892 to 2.136 & 0.148 & 1.151 & 0.743 to 1.783 & 0.528 & 1.333 & 0.838 to 2.121 & 0.224 \\
\hline Registered residence & 0.250 & 0.157 to 0.398 & $<0.01$ & 0.218 & 0.137 to 0.345 & $<0.01$ & 0.352 & 0.217 to 0.571 & 0.092 \\
\hline \multicolumn{10}{|l|}{ Medical costs } \\
\hline New Rural Cooperative Medical System & & & 0.315 & & & 0.032 & & & 0.546 \\
\hline Medical insurance & 1.475 & 1.273 to 2.138 & 0.076 & 3.762 & 0.517 to 6.319 & 0.047 & 1.545 & 0.612 to 1.941 & 0.239 \\
\hline Self-paying & 1.958 & 0.512 to 2.731 & 0.089 & 6.412 & 3.309 to 12.076 & 0.017 & 1.368 & 0.203 to 1.628 & 0.202 \\
\hline Frequency of dialysis & 0.882 & 0.735 to 1.059 & 0.180 & 0.905 & 0.750 to 1.092 & 0.296 & 0.937 & 0.772 to 1.136 & 0.507 \\
\hline Duration of haemodialysis & 1.716 & 0.782 to 3.769 & 0.178 & 1.703 & 0.700 to 4.144 & 0.240 & 3.073 & 1.387 to 6.807 & 0.063 \\
\hline Nephropathy visit before dialysis treatment & 0.110 & 0.064 to 0.189 & $<0.01$ & 0.056 & 0.033 to 0.097 & $<0.01$ & 0.249 & 0.148 to 0.420 & $<0.01$ \\
\hline Pre-established permanent vascular access & 0.242 & 0.154 to 0.381 & $<0.01$ & 0.114 & 0.063 to 0.208 & $<0.01$ & 0.225 & 0.140 to 0.362 & $<0.01$ \\
\hline Catheter type & 0.654 & 0.425 to 1.005 & 0.052 & 1.793 & 1.510 to 2.231 & 0.031 & 1.082 & 0.685 to 1.708 & 0.736 \\
\hline Catheter position & 0.521 & 0.338 to 0.804 & 0.603 & 0.067 & 0.034 to 0.130 & $<0.01$ & 0.325 & 0.204 to 0.516 & $<0.01$ \\
\hline Number of catheter insertion procedures & 1.148 & 0.888 to 1.485 & 0.292 & 1.669 & 1.276 to 2.183 & 0.086 & 2.471 & 1.818 to 3.360 & $<0.01$ \\
\hline Imaging examination after catheter insertion & 2.720 & 1.696 to 4.363 & 0.728 & 2.827 & 1.804 to 4.430 & $<0.01$ & 2.106 & 0.148 to 0.420 & 0.083 \\
\hline \multicolumn{10}{|l|}{ Catheter access vein } \\
\hline Femoral vein & & & 0.227 & & & $<0.01$ & & & 0.110 \\
\hline Internal jugular vein & 0.835 & 0.460 to 1.516 & 0.554 & 0.126 & 0.044 to 0.361 & $<0.01$ & 0.37 & 0.202 to 0.678 & 0.172 \\
\hline External jugular vein & 0.363 & 0.126 to 1.047 & 0.061 & 0.060 & 0.017 to 0.216 & $<0.010$ & 0.355 & 0.128 to 0.984 & 0.471 \\
\hline Subclavian vein & 0.572 & 0.219 to 1.495 & 0.255 & 0.094 & 0.027 to 0.332 & $<0.01$ & 0.321 & 0.117 to 0.882 & 0.282 \\
\hline Taking oral drug to prevent catheter thrombus & 0.218 & 0.125 to 0.379 & $<0.01$ & 0.100 & 0.059 to 0.168 & $<0.01$ & 0.189 & 0.089 to 0.362 & $<0.01$ \\
\hline Haemoglobin & 0.400 & 0.300 to 0.534 & 0.061 & 1.421 & 1.318 to 1.558 & $<0.01$ & 0.514 & 0.383 to 0.689 & 0.078 \\
\hline Albumin & 0.151 & 0.102 to 0.224 & $<0.01$ & 0.369 & 0.278 to 0.489 & 0.572 & 0.585 & 0.441 to 0.777 & 0.347 \\
\hline Ferritin & 1.857 & 1.375 to 2.508 & $<0.01$ & 0.96 & 0.717 to 1.286 & 0.786 & 0.898 & 0.663 to 1.218 & 0.49 \\
\hline
\end{tabular}


Table 3 Multivariate logistic regression of factors influencing the development of dialysis catheter-related complications

\begin{tabular}{|c|c|c|c|}
\hline Independent variables & OR & $95 \% \mathrm{Cl}$ & p Value \\
\hline \multicolumn{4}{|l|}{ Catheter infection } \\
\hline Age & 0.351 & 0.136 to 0.674 & 0.006 \\
\hline Registered residency & 0.25 & 0.12 to 0.52 & $<0.01$ \\
\hline Primary disease & 0.379 & 0.176 to 0.818 & 0.013 \\
\hline Educational level & 0.334 & 0.198 to 0.565 & $<0.01$ \\
\hline University & & & $<0.01$ \\
\hline Middle school & 1.224 & 0.547 to 2.736 & 0.623 \\
\hline Primary school or below & 10.757 & 3.637 to 31.817 & $<0.01$ \\
\hline Nephropathy visit before dialysis treatment & 0.22 & 0.096 to 0.502 & $<0.01$ \\
\hline Establishing long-term vascular access in advance & 0.401 & 0.193 to 0.832 & 0.014 \\
\hline Taking drugs orally to prevent catheter thrombus & 0.611 & 0.404 to 0.923 & 0.019 \\
\hline Albumin & 0.142 & 0.085 to 0.237 & $<0.01$ \\
\hline Ferritin & 2.162 & 1.412 to 3.308 & $<0.01$ \\
\hline \multicolumn{4}{|l|}{ Catheter dysfunction } \\
\hline Registered residency & 0.21 & 0.045 to 1.013 & $<0.01$ \\
\hline Taking drugs orally to prevent catheter thrombus & 0.106 & 0.041 to 0.274 & $<0.01$ \\
\hline Imaging examination after catheter insertion & 2.631 & 1.293 to 5.354 & 0.008 \\
\hline Catheter type & 3.493 & 1.358 to 8.983 & 0.009 \\
\hline \multicolumn{4}{|l|}{ Medical costs } \\
\hline Medical insurance & & & 0.004 \\
\hline New Rural Cooperative Medical System & 0.949 & 0.265 to 3.403 & 0.936 \\
\hline Self-paying & 13.416 & 2.541 to 70.827 & 0.002 \\
\hline Nephropathy visit before dialysis treatment & 0.048 & 0.018 to 0.124 & $<0.01$ \\
\hline Establishing long-term vascular access in advance & 0.025 & 0.006 to 0.109 & $<0.01$ \\
\hline Catheter on left or right & 0.024 & 0.007 to 0.080 & $<0.01$ \\
\hline \multicolumn{4}{|l|}{ Catheter access vein } \\
\hline Femoral vein & & & $<0.01$ \\
\hline Internal jugular vein & 0.029 & 0.005 to 0.179 & $<0.01$ \\
\hline External jugular vein & 0.011 & 0.001 to 0.089 & $<0.01$ \\
\hline Subclavian vein & 0.015 & 0.002 to 0.125 & $<0.01$ \\
\hline Haemoglobin & 2.276 & 1.101 to 4.749 & 0.012 \\
\hline \multicolumn{4}{|l|}{ Central vein stenosis } \\
\hline Catheterisation time & 1.827 & 1.175 to 2.841 & 0.007 \\
\hline Taking drugs orally to prevent catheter thrombus & 0.416 & 0.261 to 0.875 & 0.043 \\
\hline Nephropathy visit before dialysis treatment & 0.319 & 0.119 to 0.855 & 0.023 \\
\hline Establishing long-term vascular access in advance & 0.162 & 0.084 to 0.312 & $<0.01$ \\
\hline Left-sided or right-sided catheterisation & 0.514 & 0.268 to 0.986 & 0.045 \\
\hline Primary disease & 0.427 & 0.175 to 0.841 & 0.007 \\
\hline
\end{tabular}

advance were protective factors against catheter dysfunction $(\mathrm{OR}=0.048,95 \%$ CI 0.018 to 0.124 and $\mathrm{OR}=0.025$, $95 \%$ CI 0.006 to 0.109 , respectively). Regarding left-sided or right-sided catheterisation, the risk for catheter insertion on the right was lower than for the left $(\mathrm{OR}=0.024$, $95 \%$ CI 0.007 to 0.080 ). For catheter access veins, the femoral vein had a higher catheter dysfunction risk than other veins (internal jugular vein vs femoral vein, $\mathrm{OR}=0.029,95 \%$ CI 0.005 to 0.179 ; external jugular vein vs femoral vein, $\mathrm{OR}=0.011,95 \%$ CI 0.001 to 0.089 ; subclavian vein vs femoral vein, $\mathrm{OR}=0.015,95 \%$ CI 0.002 to $0.125)$. Haemoglobin level was also important as patients with higher levels were better able to tolerate dialysis $(\mathrm{OR}=2.276,95 \%$ CI 1.101 to 4.794$)$.

Analysis of the relationship between independent variables and central vein stenosis by logistic regression showed that there were independent relationships between central vein stenosis and the following factors: catheterisation frequency $(\mathrm{OR}=1.827,95 \%$ CI 1.175 to 2.841; the risk for patients with multiple punctures was higher), taking drugs orally to prevent catheter thrombus $(\mathrm{OR}=0.416,95 \%$ CI 1.261 to 0.875 ; the risk for patients not taking drugs was higher than for patients taking drugs), left-sided or right-sided catheterisation ( $\mathrm{OR}=0.514,95 \%$ CI 0.268 to 0.986 ; left-sided catheterisation had a higher risk than right-sided catheterisation) and primary disease (OR=0.427, 95\% CI 0.175 to 0.841 ; the risk for patients with diabetes mellitus was obviously higher than for non-diabetic patients). A nephropathy visit before dialysis treatment and establishing long-term vascular access in advance were protective factors for central vein stenosis $(\mathrm{OR}=0.319,95 \%$ CI 0.119 to 0.855 and $\mathrm{OR}=0.162,95 \%$ CI 0.084 to 0.312 , respectively). 
Complications according to type of catheter: TVC or NTHC

NTHCs were inserted 564 times and TVCs 385 times in the 865 patients included in this study, for a total of 949 insertions. NTHCs were monitored for 43051 catheterdays and TVCs for 90758 catheter-days. Serious acute complications in the 949 cases were rare. Only two patients experienced haemopneumothorax as a consequence of internal jugular vein cannulation and one patient experienced a haematoma due to femoral vein cannulation. All patients recovered fully from complications without removal of the haemodialysis catheter.

The incidence of infection related to TVCs was 5.39/ 1000 catheter-days, affecting $30.29 \%$ of patients, which was lower than that for NTHCs, which was 12.71/1000 catheter-days, affecting $44.92 \%$ of patients (both $\mathrm{p}<0.01)$; the incidence of catheter exit-site infection related to NTHCs was 9.15/1000 catheter-days, affecting $39.63 \%$ of patients, higher than the rate for TVCs, which was $5.06 / 1000$ catheter-days, affecting $15.55 \%$ of patients (both $\mathrm{p}<0.01$ ). The incidence of subcutaneous tunnel infection with TVCs was 2.73/1000 catheter-days, affecting $12.87 \%$ of patients, but cannot be compared with that of NTHCs as the latter do not require subcutaneous tunnels. The incidence of CRBSIs related to TVCs was $6.51 / 1000$ catheter-days, affecting $34.85 \%$ of patients, higher than the rate for NTHCs, which was $3.95 / 1000$ catheter-days, affecting $17.48 \%$ of patients (both $\mathrm{p}<0.01$ ). The incidence of NTHC dysfunction was $14.68 / 1000$ catheter-days, affecting $65.65 \%$ of patients, higher than the incidence of TVC dysfunction, which was $8.64 / 1000$ catheter-days, affecting $44.77 \%$ of patients (both $\mathrm{p}<0.01$ ). The incidence of NTHC thrombus was $14.87 / 1000$ catheter-days, affecting $57.72 \%$ of patients. The incidence of NTHC kinking and malposition was $4.41 / 1000$ catheter-days, affecting $25.41 \%$ of patients, which was higher than that for TVCs, which was 7.87/ 1000 catheter-days, affecting $38.87 \%$ of patients, and 2.13/1000 catheter-days, affecting $11.0 \%$ of patients (both $\mathrm{p}<0.01$ ). The incidence of TVC fibrin shells was $2.21 / 1000$ catheter-days, affecting $38.1 \%$ of patients, higher than that of NTHC fibrin shells, which was 1.67/ 1000 catheter-days, affecting $13.62 \%$ of patients ( $p=0.040$ for catheter-days; $p<0.01$ for patients). The incidence of TVC central venous stenosis was $0.79 / 1000$ catheter-days, affecting $15.28 \%$ of patients, which was higher than the incidence for NTHCs, which was 0.44/ 1000 catheter-days, affecting $3.86 \%$ of patients $(p=0.021$ for catheter-days; $p<0.01$ for patients).

\section{Complications related to catheter insertion site}

Table 4 shows the incidence of complications with TVCs according to catheter insertion site. There were no significant differences in the incidence of catheter infection among catheters inserted in the right internal

Table 4 Incidence of TVC-related complications

\begin{tabular}{|c|c|c|c|c|c|}
\hline \multirow[b]{2}{*}{$\begin{array}{l}\text { Catheter-related } \\
\text { complications }\end{array}$} & \multicolumn{5}{|l|}{ TVCs } \\
\hline & $\begin{array}{l}\text { Right internal } \\
\text { jugular vein }\end{array}$ & $\begin{array}{l}\text { Left internal } \\
\text { jugular vein }\end{array}$ & $\begin{array}{l}\text { Femoral } \\
\text { vein }\end{array}$ & $\begin{array}{l}\text { External } \\
\text { jugular vein }\end{array}$ & $\begin{array}{l}\text { Subclavian } \\
\text { vein }\end{array}$ \\
\hline \multicolumn{6}{|l|}{ Infection } \\
\hline \multicolumn{6}{|l|}{ Exit-site infection } \\
\hline$\%$ & 14.35 & 15.31 & 37.5 & 16 & 16.22 \\
\hline Times/1000 catheter-days & 4.93 & 5.06 & 10.68 & 5.26 & 5.16 \\
\hline \multicolumn{6}{|l|}{ Tunnel infection } \\
\hline$\%$ & 11.96 & 13.27 & 18.75 & 12 & 13.51 \\
\hline Times/1000 catheter-days & 2.76 & 2.68 & 3.56 & 2.72 & 2.75 \\
\hline \multicolumn{6}{|l|}{ CRBSI } \\
\hline$\%$ & 34.45 & 34.70 & 43.75 & 36 & 35.14 \\
\hline Times/1000 catheter-days & 6.69 & 6.49 & 10.08 & 6.62 & 6.42 \\
\hline \multicolumn{6}{|l|}{ Dysfunction } \\
\hline \multicolumn{6}{|l|}{ Thrombus } \\
\hline$\%$ & 39.71 & 58.16 & 62.5 & 40 & 37.84 \\
\hline Times/1000 catheter-days & 7.96 & 13.94 & 14.83 & 7.81 & 7.68 \\
\hline \multicolumn{6}{|l|}{ Fibrin shell } \\
\hline$\%$ & 38.76 & 37.76 & 37.5 & 36 & 37.84 \\
\hline Times/1000 catheter-days & 2.15 & 2.03 & 2.08 & 2.21 & 2.29 \\
\hline \multicolumn{6}{|l|}{ Malposition or kinking } \\
\hline$\%$ & 11.48 & 29.51 & 43.75 & 12 & 13.51 \\
\hline Times/1000 catheter-days & 2.07 & 3.25 & 3.26 & 2.21 & 2.19 \\
\hline \multicolumn{6}{|l|}{ Central venous stenosis } \\
\hline$\%$ & 12.44 & 27.55 & 0 & 28 & 27.03 \\
\hline Times/1000 catheter-days & 0.59 & 1.17 & 0 & 1.19 & 1.15 \\
\hline
\end{tabular}

The presence of a fibrin shell and central venous stenosis had to be demonstrated by imaging before it was included in statistical analysis. CRBSI, catheter-related bloodstream infection; TVC, tunnelled vascular catheter. 
jugular vein, left internal jugular vein, external jugular vein or subclavian vein $(\mathrm{p}=0.196$ for catheter-days; $\mathrm{p}=0.992$ for patients). The incidence of thrombosis ( $p<0.01$ for catheter-days; $p=0.017$ for patients) and the incidence of malpositioned or kinked catheters ( $p=0.024$ for catheter-days; $p=0.001$ for patients) were higher for the left internal jugular vein than the right internal jugular vein, external jugular vein and subclavian vein. The incidence of fibrin shells $(p=0.993$ for catheter-days; $p=0.999$ for patients) was not significantly different between access veins. The incidence of central venous stenosis in the right internal jugular vein was lower than in the left internal jugular vein, external jugular vein and subclavian vein $(p=0.036$ for catheterdays; $\mathrm{p}=0.004$ for patients). The incidence of femoral vein catheter infection $(\mathrm{p}<0.01$ for catheter-days; $\mathrm{p}=0.042$ for patients $)$, thrombus $(\mathrm{p}<0.01$ for catheterdays; $p=0.016$ for patients) and malpositioned or kinked catheters $(\mathrm{p}=0.036$ for catheter-days) was much higher than for other access veins. Inferior vena cava stenosis caused by femoral venipuncture was not seen.

Table 5 shows the incidence of infection ( $p=0.972$ for catheter-days; $\mathrm{p}=0.998$ for patients) and venous thrombosis ( $p=0.991$ for catheter-days; $p=0.988$ for patients) in the right internal jugular vein. Left internal jugular vein and subclavian vein showed no significant differences. The incidence of infection (both $\mathrm{p}<0.01$ ) and venous thrombosis (both $\mathrm{p}<0.01$ ) in the femoral vein were much higher than that in the internal jugular vein and subclavian vein, but the incidence of fibrin sheath in the femoral vein were the lowest (both $\mathrm{p}<0.01$ ). The incidence of malpositioned or kinked catheters was highest in the femoral vein $(p<0.01$ for catheter-days; $p=0.01$ for patients), while the incidence of malpositioned or kinked catheters was higher in the left internal jugular vein than in the right internal jugular vein and subclavian vein $(p<0.01$ for catheter-days; $p=0.080$ for patients). The incidence of central venous stenosis in the (left-sided and right-sided) internal jugular vein were not significantly different $(p=0.310$ for catheterdays; $p=0.343$ for patients). Inferior vena cava stenosis was not seen with femoral vein NTHCs.

\section{Complications related to make of catheter}

The incidence rates of catheter type-related complications are presented in table 6 . The incidence of infection ( $p=0.976$ for catheter-days; $p=0.985$ for patients) and fibrin shell formation ( $\mathrm{p}=0.963$ for catheter-days; $\mathrm{p}=1.000$ for patients) were not significantly different among the four types of TVCs; the highest incidence of catheter thrombosis was seen with HemoSplit catheters and the lowest incidence with Palindrome catheters ( $p<0.01$ for catheter-days; $p=0.002$ for patients). The incidence of kinking or malposition using Permcath and

Table 5 Incidence of NTHC-related complications

\begin{tabular}{|c|c|c|c|c|}
\hline \multirow[b]{2}{*}{$\begin{array}{l}\text { Catheter-related } \\
\text { complications }\end{array}$} & \multicolumn{4}{|l|}{ NTHCs } \\
\hline & $\begin{array}{l}\text { Right internal } \\
\text { jugular vein }\end{array}$ & $\begin{array}{l}\text { Left internal } \\
\text { jugular vein }\end{array}$ & $\begin{array}{l}\text { Femoral } \\
\text { vein }\end{array}$ & $\begin{array}{l}\text { Subclavian } \\
\text { vein }\end{array}$ \\
\hline \multicolumn{5}{|l|}{ Infection } \\
\hline \multicolumn{5}{|l|}{ Exit-site infection } \\
\hline$\%$ & 36.7 & 36.2 & 48.37 & 35.48 \\
\hline Times/1000 catheter-days & 8.79 & 8.54 & 19.66 & 8.85 \\
\hline \multicolumn{5}{|l|}{ Tunnel infection } \\
\hline$\%$ & - & - & - & - \\
\hline Times/1000 catheter-days & - & - & - & - \\
\hline \multicolumn{5}{|l|}{ CRBSI } \\
\hline$\%$ & 16.51 & 17.39 & 29.77 & 17.74 \\
\hline Times/1000 catheter-days & 3.57 & 3.61 & 9.93 & 3.75 \\
\hline \multicolumn{5}{|l|}{ Dysfunction } \\
\hline \multicolumn{5}{|l|}{ Thrombus } \\
\hline$\%$ & 58.7 & 59.42 & 78.6 & 58.06 \\
\hline Times/1000 catheter-days & 14.74 & 15 & 29.98 & 14.79 \\
\hline \multicolumn{5}{|l|}{ Fibrin shell } \\
\hline$\%$ & 13.76 & 14.49 & 4.19 & 14.53 \\
\hline Times/1000 catheter-days & 1.53 & 1.9 & 0.39 & 1.69 \\
\hline \multicolumn{5}{|l|}{ Malposition or kinking } \\
\hline$\%$ & 24.31 & 37.68 & 45.12 & 24.19 \\
\hline Times $/ 1000$ catheter-days & 4.37 & 10.06 & 19.86 & 4.86 \\
\hline \multicolumn{5}{|l|}{ Central venous stenosis } \\
\hline$\%$ & 3.67 & 4.35 & 0 & 8.06 \\
\hline Times/1000 catheter-days & 0.45 & 0.27 & 0 & 0.62 \\
\hline
\end{tabular}




\begin{tabular}{|c|c|c|c|c|c|c|c|c|}
\hline & TVC type & & & & NTHC type & & & \\
\hline Catheter-related complications & Palindrome & Permcath & Cannon II Plus & HemoSplit & ARROW & DIALL & ABEL & TYCO \\
\hline \multicolumn{9}{|l|}{ Infection } \\
\hline$\%$ & 15.69 & 15.38 & 15.24 & 16.09 & 36.08 & 36.2 & 35.97 & 35.58 \\
\hline Times/1000 catheter-days & 5.12 & 5.08 & 4.97 & 5.17 & 8.96 & 9.08 & 9.05 & 8.82 \\
\hline \multicolumn{9}{|l|}{ Tunnel infection } \\
\hline$\%$ & 12.75 & 13.19 & 12.38 & 13.79 & - & - & - & - \\
\hline Times/1000 catheter-days & 2.74 & 2.7 & 2.75 & 2.83 & - & - & - & - \\
\hline \multicolumn{9}{|l|}{ CRBSI } \\
\hline$\%$ & 35.29 & 35.16 & 35.24 & 35.63 & 17.09 & 17.18 & 17.27 & 17.31 \\
\hline Times/1000 catheter-days & 6.53 & 6.43 & 6.5 & 6.68 & 3.48 & 3.54 & 4.05 & 3.65 \\
\hline \multicolumn{9}{|l|}{ Catheter dysfunction } \\
\hline \multicolumn{9}{|l|}{ Thrombus } \\
\hline$\%$ & 22.55 & 38.46 & 39.05 & 49.42 & 57.59 & 57.67 & 57.55 & 57.69 \\
\hline Times/1000 catheter-days & 5.32 & 8.76 & 7.31 & 9.8 & 15.01 & 15.03 & 14.51 & 14.86 \\
\hline \multicolumn{9}{|l|}{ Fibrin shell } \\
\hline$\%$ & 38.24 & 38.46 & 38.1 & 37.93 & 13.92 & 14.11 & 13.67 & 13.46 \\
\hline Times/1000 catheter-days & 2.25 & 2.06 & 2.1 & 2.07 & 1.49 & 1.53 & 1.6 & 1.51 \\
\hline \multicolumn{9}{|l|}{ Malposition or kinking } \\
\hline$\%$ & 18.63 & 18.68 & 8.57 & 10.34 & 31.01 & 30.67 & 30.22 & 30.77 \\
\hline Times/1000 catheter-days & 2.73 & 2.66 & 1.47 & 1.51 & 8.54 & 8.52 & 8.29 & 8.19 \\
\hline \multicolumn{9}{|l|}{ Central venous stenosis } \\
\hline$\%$ & 14.71 & 15.38 & 3.81 & 14.94 & 3.8 & 4.91 & 4.32 & 3.85 \\
\hline Times/1000 catheter-days & 0.75 & 0.84 & 0.24 & 0.88 & 0.5 & 0.64 & 0.57 & 0.5 \\
\hline $\mathrm{Kt} / \mathrm{V}$ & $1.40 \pm 0.73$ & $1.39 \pm 0.19$ & $1.38 \pm 0.13$ & $1.38 \pm 0.17$ & $1.06 \pm 0.07$ & $1.05 \pm 0.07$ & $1.06 \pm 0.13$ & $1.06 \pm 0.08$ \\
\hline
\end{tabular}


Palindrome catheters was not significantly different ( $p=0.857$ for catheter-days; $p=0.992$ for patients) but was higher than for Cannon II Plus and HemoSplit catheters ( $\mathrm{p}<0.01$ for catheter-days; $\mathrm{p}=0.030$ for patients). The incidence of central venous stenosis with Cannon II Plus catheters was lower than with other catheters $(\mathrm{p}=0.025$ for catheter-days; $p=0.028$ for patients). Urea clearance index $(\mathrm{Kt} / \mathrm{V})$ values were higher in patients using Palindrome catheters than any of the other three types of TVCs, but the difference was not statistically significant $(\mathrm{p}=0.990)$.

The incidence of catheter infection $(\mathrm{p}=0.971$ for catheter-days; $p=1.000$ for patients) and the incidence of central venous stenosis $(\mathrm{p}=0.963$ for catheter-days; $\mathrm{p}=0.961$ for patients) was not significantly different among the four types of NTHCs. The incidences of thrombus $(p=0.988$ for catheter-days; $p=1.000$ for patients), catheter kinking or malposition $(p=0.991$ for catheter-days; $\mathrm{p}=0.999$ for patients) and fibrin shell formation ( $p=0.997$ for catheter-days; $p=0.999$ for patients) was also not significantly different between the four types of NTHCs. Kt/V values were not significantly different between the four types of NTHCs $(p=0.965)$.

\section{DISCUSSION}

The objective of this study was to investigate the incidence of and risk factors for catheter-related complications in different districts and populations in Henan Province in China. The overall incidence was 7.74/1000 catheter-days, affecting $38.61 \%$ of patients, for catheter infection, 10.58/1000 catheter-days, affecting $56.65 \%$ of patients, for catheter dysfunction, and $0.68 / 1000$ catheter-days, affecting $8.79 \%$ of patients, for central vein stenosis. Multivariate analysis showed many different factors were associated with the risk of catheter infection, catheter dysfunction and central vein stenosis.

Previous studies showed that the rate of CRI was about 3.8-6.5/1000 catheter-days. ${ }^{13}{ }^{14}$ However, the rate of CRI was higher in the present study. There may be a few reasons for this higher rate. The first are the characteristics of the patients. The patients in this study were relatively older (mean age: $49.53 \pm 18.42$ years) and so were more likely to have age-related multiple organ function damage, malnutrition, impaired immunity and frailty, all of which could increase the risk of catheter infection. Diabetes was the most frequent primary disease (27.3\%), and long-term diabetes is associated with peripheral vascular disorders, preventing AVF insertion and requiring the use of catheters for dialysis. The risk of CRI was increased by $60 \%$ in patients with diabetes. ${ }^{15}$ Patients with a higher level of education may be more concerned about the severity of their disease and understand better how to look after dialysis catheters. Patients living in urban settings are generally better educated than those living in the countryside. However, most of the patients in the present study were from rural areas $(57.5 \%)$ or were educated to middle school level or below.
Dialysis imposes a heavy financial burden on patients. In the present study, some patients had medical insurance under the New Rural Cooperative Medical System $(44.6 \%)$ but some had no medical insurance at all $(18.5 \%)$.

The overall infection rate in the NTHC group was significantly higher than in the TVC group, which is supported by previous findings. ${ }^{14}$ Indeed, the higher rate of CRBSI in the TVC group (1052.28 \pm 371.26 days) compared with the NTHC group (136.2 \pm 67.4 days) might have been due to the longer duration of catheter placement. Oliver $e t a l^{14}$ showed that the duration of catheter placement was a high risk factor for CRI. Weijmer $e a^{16}$ suggested that for patients needing an NTHC for more than 2 weeks, the catheter should be changed to a TVC to reduce the risk of infection. Longer catheter placement time was generally associated with a low patient educational level (suggesting they were not fully aware of the severity of the disease). In the present study, $85.5 \%$ of patients underwent two or more procedures to obtain vascular access.

Catheter dysfunction is characterised by poor blood flow. Previous studies showed that $98 \%$ of catheter dysfunction occurring within 2 weeks of insertion was caused by either a catheter embolism or a fibrin shell. ${ }^{4}$ Suhocki et $a l^{17}$ reported that among 163 patients with catheter dysfunction, $74.2 \%$ had a catheter embolism and $23.3 \%$ had a fibrin shell. Napalkov et $a l^{18}$ investigated 3213 patients using dialysis catheters and found that the incidence of thrombosis was 8.6/1000 catheterdays. In the present study, the overall incidence of catheter dysfunction was 10.58/1000 catheter-days for all patients, 8.64/1000 catheter-days for TVCs (which was in accordance with previous studies) and 12.86/1000 catheter-days for NTHCs (which was substantially higher than in previous studies). NTHC catheter type was a risk factor for catheter dysfunction in this study. Hyperlipidaemia, arteriosclerosis, blood disease and cancer can induce hypercoagulability. ${ }^{19}$ In addition, patients with uraemia generally have high homocysteine levels and microinflammation, which can increase thrombogenesis. ${ }^{19}$ Too many catheter insertions could damage the vascular wall, and exposure of the endothelium could promote platelet adhesion and activate the endogenous coagulation pathway, which could be further aggravated by local disturbance of haemodynamics after catheter insertion. ${ }^{20}$ However, we did not find the time taken for catheter insertion was associated with catheter dysfunction in this study. Fibrin shells can develop with central venous catheters and can induce both catheter dysfunction and a series of complications including infection, thrombogenesis, the necessity of catheter removal and pulmonary embolism. ${ }^{21}{ }^{22}$ Previous studies showed that the incidence of fibrin shells was generally high. In a study by Oliver $e t a l^{23}$ indwelling catheters had a fibrin shell in $70 \%$ of patients. Schon and Whittman ${ }^{24}$ also showed that $38 \%$ of patients with catheter dysfunction had a fibrin shell. 
In the present study, the incidence of fibrin shells was $3.69 / 1000$ catheter-days, affecting $2.18 \%$ of catheters, in the NTHC group, and 3.81/1000 catheter-days, affecting $2.41 \%$ of catheters, in the TVC group, which values were much lower than in previous studies. Although drug treatment or fibrin removal could restore partial catheter function in some cases, disadvantages include the high recurrence rate after drug treatment and the high cost of fibrin removal. Consequently, it was cheaper to replace the dialysis catheter than perform further imaging, which may have caused the rate of fibrin shell formation to be underestimated.

The incidence of central vein stenosis can be as high as $25-40 \%{ }^{25}$ Several studies also showed that $95 \%$ of patients with central vein stenosis had a history of an indwelling catheter, while only $13.6 \%$ of patients without a history of an indwelling catheter developed central vein stenosis, and that repeated catheter insertions could increase by threefold the incidence of central vein stenosis in dialysis patients. ${ }^{26}$ Following catheter insertion, mechanical injuries to the vascular endothelium, secondary activation of platelet and coagulation pathways, changes in haemodynamics and extrinsic compression of tissues surrounding the catheter contributed to the development of central vein stenosis. ${ }^{27}$ Angiography is the gold standard for the diagnosis of central vein stenosis. $^{28}$ In the present study, the overall incidence rate of central vein stenosis $(0.68 / 1000$ catheter-days) was relatively low, which could be due to a low rate of diagnosis. Indeed, only patients with severe symptoms (including severe facial swelling, limb swelling, limb pain and intracranial hypertension) were examined by angiography, while most patients with mild or no symptoms were not investigated due to high medical costs or insufficient awareness by clinicians. The incidence of central vein stenosis was significantly higher in patients using TVCs $(0.79 / 1000$ catheter-days) compared those using NTHCs $(0.44 / 1000$ catheter-days, $\mathrm{p}<0.05)$, which might be associated with damage to the vascular wall caused by the long duration of TVC placement. Diabetes is an important risk factor for central vein stenosis, which could be caused by the long-term affects of high glucose, chronic inflammation and lipid metabolism disorders, increasing the risk of angiosclerosis, vascular wall thickening, reduced elasticity, thrombogenesis and stenosis in diabetic patients on dialysis. ${ }^{29}$

In this study, four different types of TVCs and four different types of NTHCs were used in the 14 participating hospitals. Since no specific coating was applied to any of the TVCs to prevent infection and thrombosis, and since the duration of placement of these four types of TVCs was similar, no significant difference in the incidence rates of catheter infection was found among the four types of TVCs. However, the incidence of catheter embolism was significantly higher with the HemoSplit catheter compared with the other types, while patients using the Palindrome catheter had the lowest incidence of catheter embolism, which might be associated with the different designs of the side holes at the tip of the catheters. The side holes in the Palindrome catheter are quite large rectangles made by laser notching, which are relatively difficult for thrombi to adhere to, ${ }^{30}$ while the side holes in the HemoSplit catheter are quite small round holes which thrombi can easily adhere to. The texture and cuff rings are identical in the Palindrome and Permcath catheters, which also had significantly higher the rates of catheter kinking and malposition compared to the other two types of TVCs. The specific 'retrograde design' of the Cannon II Plus catheter ${ }^{31}$ ensures that the cuff ring is fixed above the clavicle, while the cuff ring of the HemoSplit catheter can be embedded in adjacent tissue. Therefore, these two catheters had a lower risk of kinking and malposition. The incidence rate of central vein stenosis with the Cannon II Plus catheter was the lowest among all four types of TVCs, which could be related to the fact that the tip of the catheter was placed in the right atrium so the affect on the superior vena cava was relatively small. There were no significant differences in the incidence of complications between the four different types of NTHCs used in this study.

These results suggest that healthcare policies for patients with chronic renal disease should be modified as follows: (1) a nephrologist should periodically interview the patient with ESRD, and educate them about haemodialysis and vascular access; (2) strict early referral procedures should be implemented to avoid delayed diagnosis and treatment of ESRD due to lack of general practitioner expertise; (3) health administrative departments should evaluate the quality of haemodialysis based on the ratio of patients using catheters in the centres; and (4) haemodialysis centres should set up vascular access teams consisting of a nephrologist, haemodialysis experts, haemodialysis nurse specialist, vascular surgeon, interventional radiologist and maintenance technician to ensure optimum treatment of the haemodialysis patient.

This study has several limitations. First, there is some residual confounding from unmeasured variables such as medical treatment, patient nutritional status, biochemical indices and psychosocial factors. Second, Henan Province covers a large area with a large population and has widely varying cultural practices and economic and healthcare levels among the different districts. Since all 14 participating hospitals were from districts with a relatively high economic level, the epidemiological features of catheter-related complications could be different in remote areas or in districts with a lower economic level. Therefore, the incidence of catheter-related complications could be underestimated in the present study.

In conclusion, patients receiving dialysis in Henan Province had a high rate of dialysis catheter use with long duration of temporary catheter placement which resulted in a high incidence of catheter-related complications. Therefore, the healthcare administration should 
develop policies to assess patients early, ideally before dialysis is required, to allow permanent vascular access to be established in advance as soon as possible.

Contributors KW and ZL: conception and design; KW, PW, XLi, XLu and ZL: acquisition of data or analysis and interpretation of data; KW and ZL: drafting the manuscript and revising it critically for important intellectual content. All authors gave final approval of the version to be published.

\section{Funding None.}

Competing interests None declared.

Patient consent Obtained.

Ethics approval The study was approved by the ethics committee of The First Affiliated Hospital, Zhengzhou University, Institute of Nephrology, Zhengzhou University, Zhengzhou 450052, Henan Province, China.

Provenance and peer review Not commissioned; externally peer reviewed.

Data sharing statement $A$ technical appendix, statistical code and dataset are available from the corresponding author at the Dryad digital repository, which will provide a permanent, citable and open access repository for the dataset.

Open Access This is an Open Access article distributed in accordance with the Creative Commons Attribution Non Commercial (CC BY-NC 4.0) license, which permits others to distribute, remix, adapt, build upon this work noncommercially, and license their derivative works on different terms, provided the original work is properly cited and the use is non-commercial. See: http:// creativecommons.org/licenses/by-nc/4.0/

\section{REFERENCES}

1. Stevens PE, Levin A. Evaluation and management of chronic kidney disease: synopsis of the kidney disease: improving global outcomes 2012 clinical practice guideline. Annals of Internal Medicine 2013;158:825-30.

2. Ravani P, Palmer SC, Oliver MJ, et al. Associations between hemodialysis access type and clinical outcomes: a systematic review. J Am Soc Nephrol 2013;24:465-73.

3. Allon M, Daugirdas J, Depner TA, et al. Effect of change in vascular access on patient mortality in hemodialysis patients. Am J Kidney Dis 2006;47:469-77.

4. [No authors listed]. III. NKF-K/DOQI Clinical Practice Guidelines for Vascular Access: update 2000. Am J Kidney Dis 2001;37:S137-81.

5. Foley RN, Collins AJ. The USRDS: what you need to know about what it can and can't tell us about ESRD. Clin J Am Soc Nephrol 2013;8:845-51.

6. Lacson $\mathrm{E} \mathrm{Jr}$., Lazarus JM, Himmelfarb J, et al. Balancing fistula first with catheters last. Am J Kidney Dis 2007;50:379-95.

7. Allon M. Dialysis catheter-related bacteremia: treatment and prophylaxis. Am J Kidney Dis 2004:44:779-91.

8. Lee T, Barker J, Allon M. Tunneled catheters in hemodialysis patients reasons and subsequent outcomes. Am J Kidney Dis 2005;46:501-8.

9. Shapiro MA, Johnson M, Feinstein SB. A retrospective experience of right atrial and superior vena caval thrombi diagnosed by transesophageal echocardiography. J Am Soc Echocardiogr 2002;15:76-9.

10. US Renal Data System. USRDS 2009 Annual Data Report: Atlas of Chronic Kidney Disease and End-Stage Renal Disease in the United States. Bethesda: National Institute of Health, National Institute of Diabetes and Digestive and Kidney Disease, 2009.
11. Brenner L, Singh AK, Campbell D, et al. Associations between demographic factors and provider structures on cost and length of stay for hemodialysis patients with vascular access failure. Clin J Am Soc Nephrol 2006;1:455-61.

12. Lee T, Mokrzycki M, Moist L, et al. Standardized definitions for hemodialysis vascular access. Semin Dial 2011;24:515-24.

13. Kairaitis LK, Gottlieb T. Outcome and complications of temporary haemodialysis catheters. Nephrol Dial Transplant 1999;14: 1710-14.

14. Oliver MJ, Callery SM, Thorpe KE, et al. Risk of bacteremia from temporary hemodialysis catheters by site of insertion and duration of use: a prospective study. Kidney Int 2000;58:2543-5.

15. Saxena AK, Panhotra BR, Chopra R. Advancing age and the risk of nasal carriage of Staphylococcus aureus among patients on long-term hospital-based hemodialysis. Ann Saudi Med 2004;24:337-42.

16. Weijmer MC, Vervloet MG, ter Wee PM. Compared to tunnelled cuffed haemodialysis catheters, temporary untunnelled catheters are associated with more complications already within 2 weeks of use. Nephrol Dial Transplant 2004;19:670-7.

17. Suhocki PV, Conlon PJ Jr, Knelson MH, et al. Silastic cuffed catheters for hemodialysis vascular access: thrombolytic and mechanical correction of malfunction. Am J Kidney Dis 1996;28:379-86.

18. Napalkov P, Felici DM, Chu LK, et al. Incidence of catheter-related complications in patients with central venous or hemodialysis catheters: a health care claims database analysis. BMC Cardiovasc Disord 2013;13:86.

19. Platt A. Can you recognize a patient at risk for a hypercoagulable state? JAAPA 2008;21:20-6.

20. Xiang DZ, Verbeken EK, Van Lommel AT, et al. Intimal hyperplasia after long-term venous catheterization. Eur Surg Res 2000;32:236-45.

21. Faintuch S, Salazar GM. Malfunction of dialysis catheters: management of fibrin sheath and related problems. Tech Vasc Interv Radiol 2008;11:195-200.

22. Bona RD. Thrombotic complications of central venous catheters in cancer patients. Semin Thromb Hemost 1999;25:147-55.

23. Oliver MJ, Mendelssohn DC, Quinn RR, et al. Catheter patency and function after catheter sheath disruption: a pilot study. Clin J Am Soc Nephrol 2007;2:1201-6

24. Schon D, Whittman D. Interventional nephrology and dialysis: managing the complications of long-term tunneled dialysis catheters. Semin Dial 2003;16:314-22.

25. Kundu S. Central venous disease in hemodialysis patients: prevalence, etiology and treatment. J Vasc Access 2010; 11:1-7.

26. MacRae JM, Ahmed A, Johnson N, et al. Central vein stenosis: a common problem in patients on hemodialysis. ASAIO J 2005;51:77-81.

27. Yevzlin AS. Hemodialysis catheter-associated central venous stenosis. Semin Dial 2008;21:522-7.

28. Agarwal AK, Haddad NJ, Khabiri H. How should symptomatic centra vein stenosis be managed in hemodialysis patients? Semin Dial 2014;27:278-81.

29. Cheng $\mathrm{Y}, \mathrm{Cui} \mathrm{T}, \mathrm{Fu} \mathrm{P}$, et al. Dyslipidemia is associated with tunneled-cuffed catheter-related central venous thrombosis in hemodialysis patients: a retrospective, multicenter study. Artif Organs 2013;37:E155-61.

30. Spector M, Mojibian H, Eliseo D, et al. Clinical outcome of the Tal Palindrome chronic hemodialysis catheter: single institution experience. J Vasc Interv Radiol 2008;19:1434-8.

31. Davanzo WJ. Efficacy and safety of a retrograde tunneled hemodialysis catheter: 6-month clinical experience with the Cannon Catheter chronic hemodialysis catheter. J Vasc Access 2005;6:38-44. 